\title{
Optical coherence tomography evaluating unstable coronary plaques: A case report
}

\author{
J inchuan Yan, Cuiping Wang, Wei Yuan \\ Department of Cardiology, Affiliated Hospital of Jiangsu University, Zhenjiang, Jiangsu Province, P. R. China. \\ Correspondence: Jin-Chuan Yan. Address: Affiliated Hospital of Jiangsu University, Zhenjiang, Jiangsu Province, P. R. \\ China. Email: yanjinchuan@hotmail.com
}

Received: August 27, 2014

DOI : $10.5430 /$ crim.v2n1p77

\begin{abstract}
Unstable plaque is the main cause of ACS. However, it is difficult to find in some cases. We report a case in which conventional angiography and IVUS were unable to identify the ruptured plaque that caused the condition in a 72-year-old man. He has been suffered from accelerated angina at rest. Angiography and IVUS did not find culprit lesion. However, a ruptured thin-cap fibroatheroma plaque was found in right coronary using OCT. The symptom was disappeared after one stent coving the lesion. The case indicates that OCT is a new intravascular imaging modality that allows clear visualization of vulnerable plaques.
\end{abstract}

\section{Keywords}

Acute coronary syndrome, Optical coherence tomography, Ruptured plaque

\section{I ntroduction}

In vitro pathological studies have demonstrated that most cases of acute coronary syndrome (ACS) result from sudden luminal narrowing, which is caused by thrombosis based on plaque rupture, erosion, and superficial calcified nodule. Among these three different pathologies, plaque rupture is the most frequent (55\%-60\%). Plaque rupture is a lesion comprising a large necrotic core with a ruptured thin fibrous cap. However, the culprit lesions from ruptured plaques are difficult to identify. We present a case in which conventional coronary angiography and IVUS failed to identify the lesion causing the condition, and optical coherence tomography (OCT) was used to obtain clear images that helped confirm the diagnosis.

\section{Case report}

A 72-year-old male visited our center because of accelerated angina at rest on 16 April 2013. He underwent percutaneous coronary intervention with three drug-eluting stents in the left anterior descending (LAD) artery eight months prior to his visit. His physical examination and chest X-ray results were normal. ECG showed a q/Q wave in II, III, and avF leads and inverted T wave in V5-6 (see Figure 1). However, his cardiac troponin I level was $0.20 \mathrm{ng} / \mathrm{ml}$ (the normal value was $<0.04 \mathrm{ng} / \mathrm{ml}$ ). Transthoracic echocardiography showed $50 \%$ left ventricular ejection fraction (EF) and weak inferior wall motion. Drug therapy, including anti-platelet agents, platelet glycoprotein IIb/IIIa receptor antagonist, and low- 
molecular-weight heparin, had no effects. He underwent coronary angiography on 17 April 2013. The angiogram showed that LAD stents were normal (see Figure 2A). The circumflex artery showed total occlusion similar to the last procedure, which was performed eight months ago (see Figure 2B). A large right coronary artery with a slight stenotic lesion in the proximal artery was found (see Figure $2 \mathrm{C}$ and 2D). The patient underwent both IVUS and OCT of the LAD and right coronary. IVUS images were acquired using a 20-50 MHz imaging Eagle Eye Gold catheter (Volcano, Rancho Cordova, CA, USA). IVUS images showed that the right coronary stenotic lesion had a false lumen. The plaque burden was $72.6 \%$, and the minimum lumen area was $5.8 \mathrm{~mm}^{2}$ (see Figure 3A). Then, OCT images have been acquired using a nonocclusive technique with the C7XR system (DragonFly catheter and C7XR, LightLab Imaging). The artery was cleared of blood by continuous flushing with iodixanol 320 (Visipaque, GE Health Care, Cork, Ireland) at a flow rate of $3.0 \mathrm{ml} / \mathrm{s}$. Thus, a rupture with thin-cap fibroatheroma plaque was found in the lesion by OCT. A lesion with a $0.70 \mu \mathrm{m}$-thick discontinuous fibrous cap, lipid-rich plaque, and intimal tear was acquired (see Figure 3B to 3F). The lumen area from the IVUS was sufficient for the right coronary artery, but an unstable and ruptured coronary plaque may cause acute total occlusion in this situation. Therefore, we placed a $3.5 \mathrm{~mm} \times 18 \mathrm{~mm}$ Partnar stent covering the ruptured plaque (see Figure 4A). OCT images showed the stent malapposition. The length between the stent and intima was $0.44 \mathrm{~mm}$ (see Figure 4B). Post-dilation was performed with a $4.0 \mathrm{~mm} \times 12 \mathrm{~mm}$ non-compliant balloon (see Figure 4C). Coronary angiography yielded a satisfactory result (see Figure 4D). The patient continued to take medication, including aspirin, clopidogrel, and simvastatin, and the angina symptoms disappeared. He was discharged from the hospital $3 \mathrm{~d}$ after the procedure. As of this writing (15 months after stenting), the patient was asymptomatic.

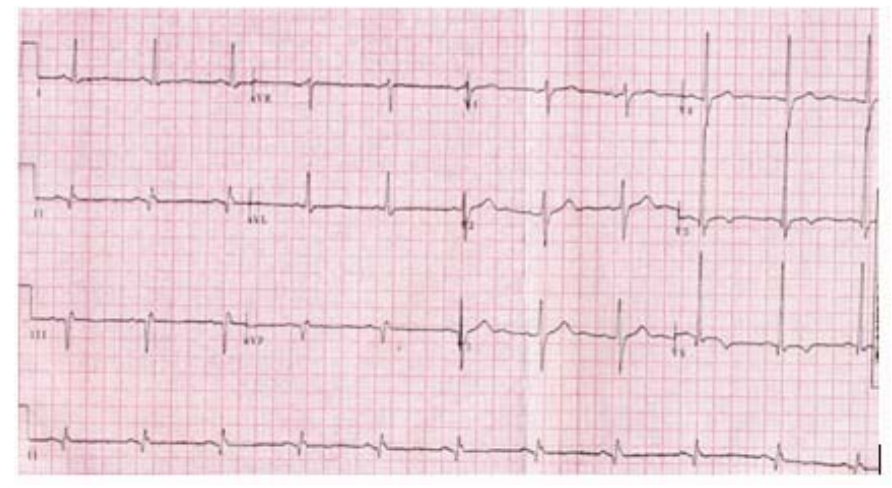

Figure 1. The ECG showed q/Q wave in II, III, avF leads and inverted T wave in V5-6

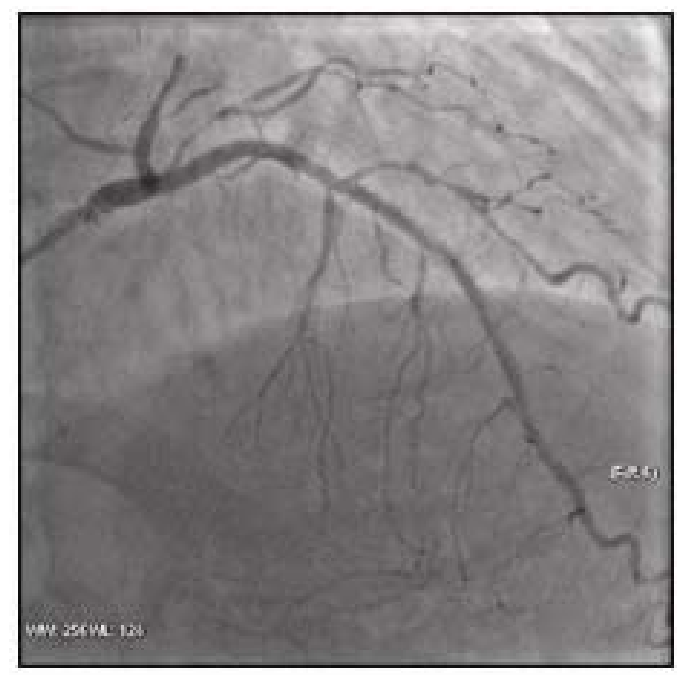

Figure 2A. LAD stents is normal

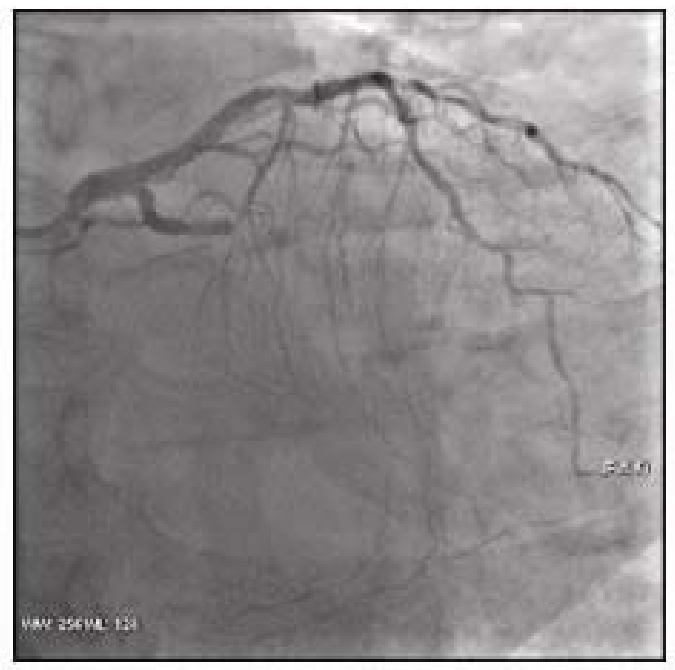

Figure 2B. Circumflex total occlusion 


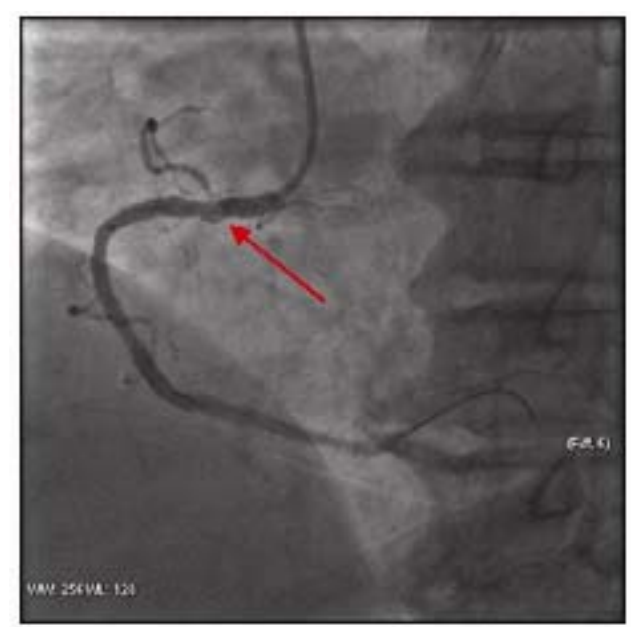

Figure 2C. Right coronary artery (RCA) with a slight stenosis lesion in the proximal

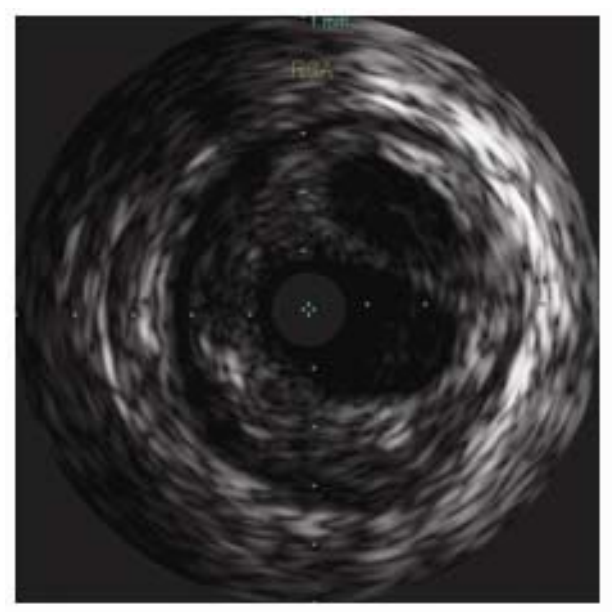

Figure 3A. IVUS showing the stenosis lesion in the proximal of RCA

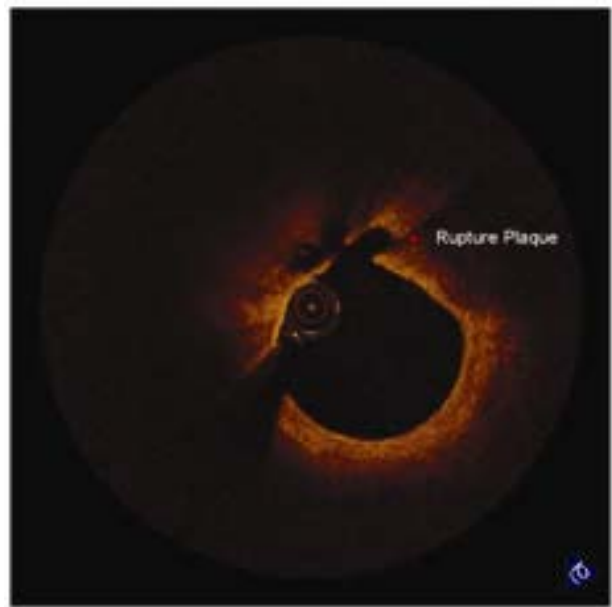

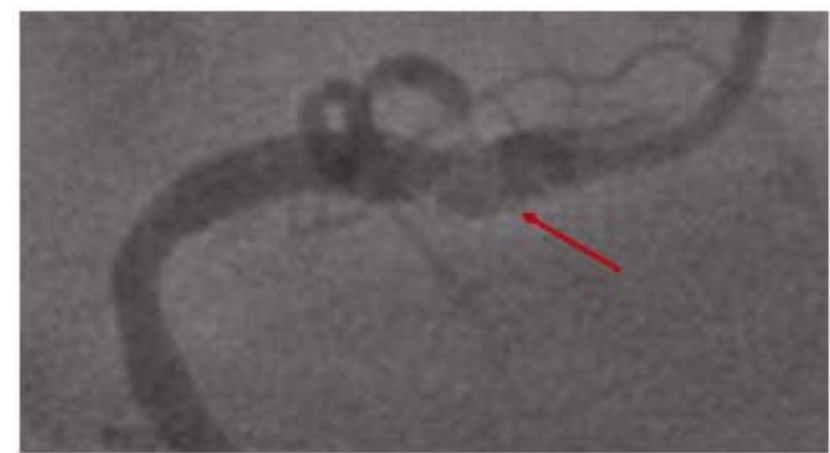

Figure 2D. Enlarged stenosis lesion in the proximal of RCA

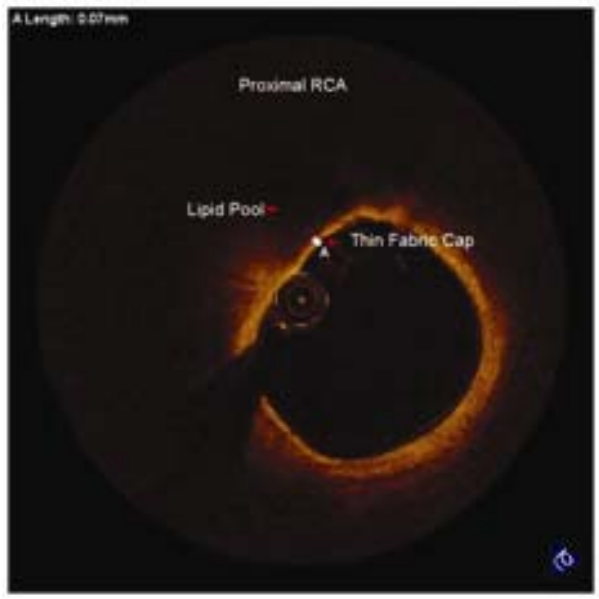

Figure 3B. OCT showing the thin fibrous cap with lipid rich plaque

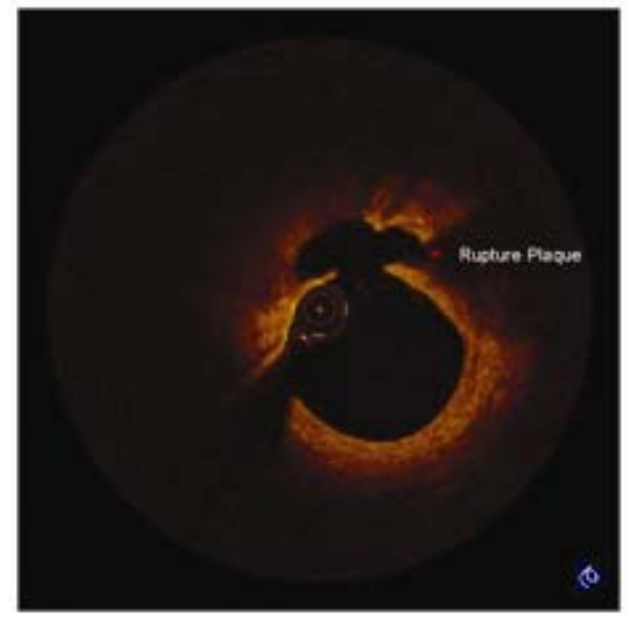

Figure 3C (left) and 3D (right). OCT showing the ruptured plaque 

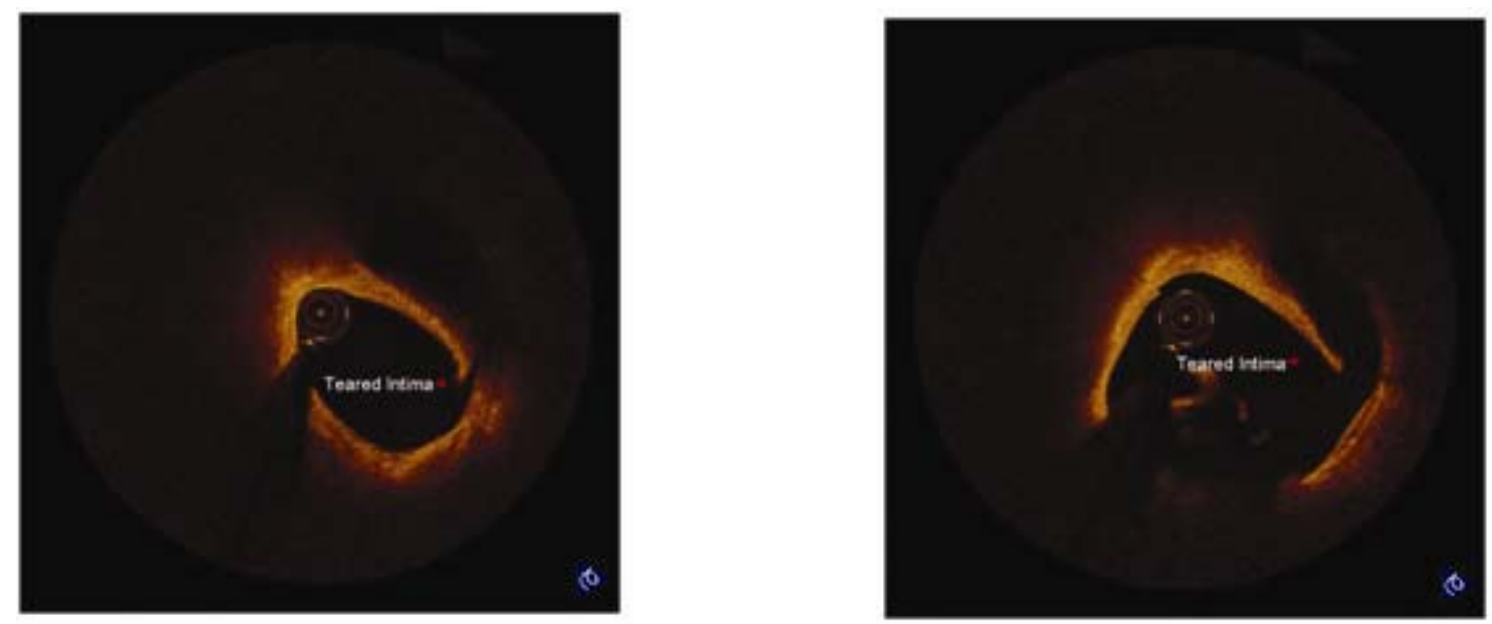

Figure 3E (left) and 3F (right). OCT showing the teared intima

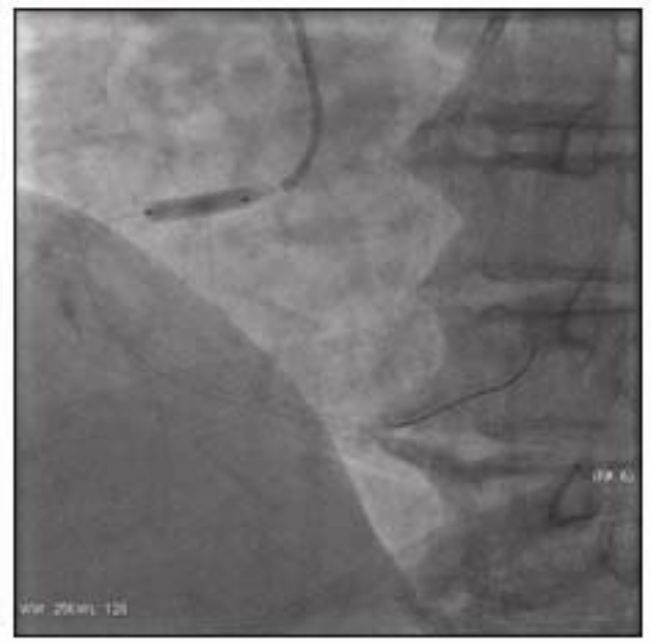

Figure 4A. The stent opening process in the rupture plaque lesion

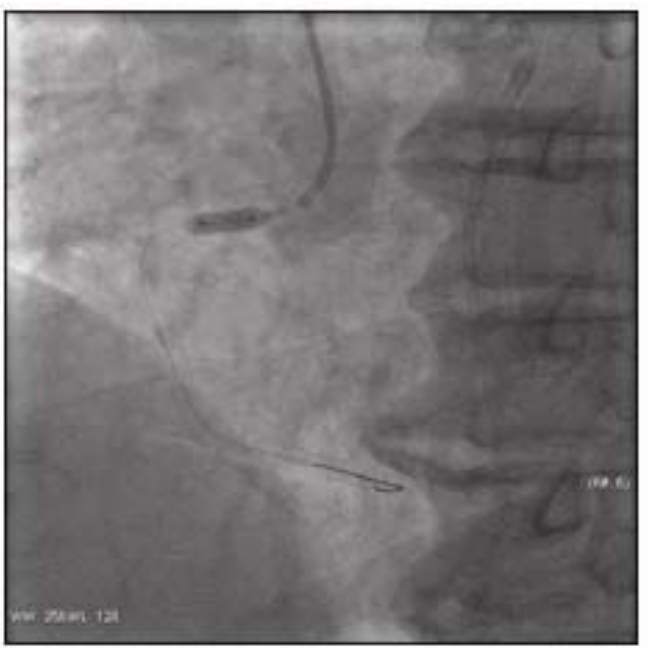

Figure 4C. Post-dilated with a balloon

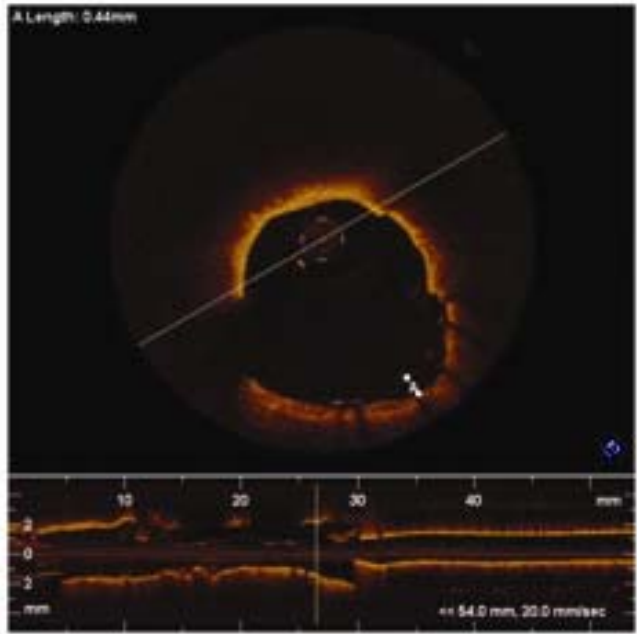

Figure 4B. OCT showing the stent malapposition

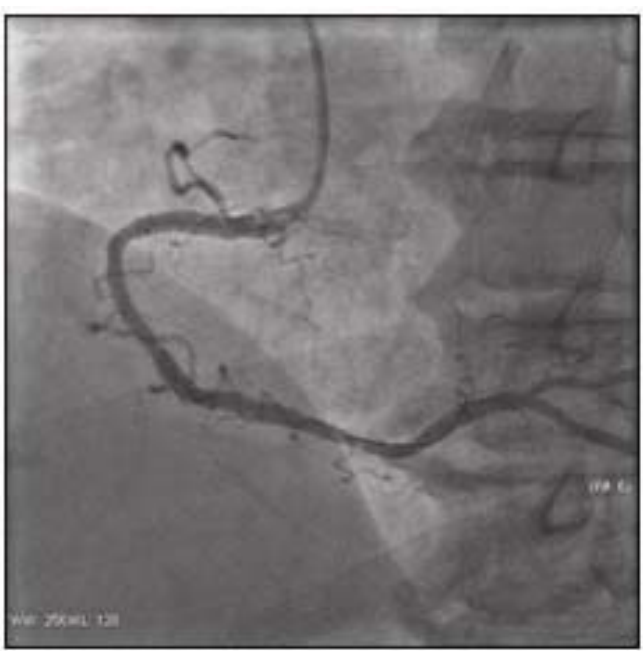

Figure 4D. The result of last procedure 


\section{Discussion}

IVUS is the most widely used intracoronary imaging technique in routine practice. It is derived using ultrasound at a frequency of 20-40 MHz, which provides an axial resolution of 100-200 $\mu \mathrm{m}$ and lateral resolution of around $250 \mu \mathrm{m}$. Compared with simple contrast angiography, IVUS can assess stent apposition and expansion after implantation and determine the morphology of atherosclerotic plaques (lipid-rich, calcified, and fibrous). IVUS is three times more powerful in detecting calcifications than angiography, and has a sensitivity and specificity of $89 \%$ and $97 \%$, respectively. These features of IVUS enable the identification of microscopic changes in the size of atheromas and assessment of their limits and surface. IVUS can be used to determine the histological composition of atherosclerotic plaques (calcium, lipids, fibrosis, and necrotic core). IVUS can measure the artery wall and its layers, enabling precise selection of stent diameter and length. These properties are useful for visualizing deep structures, but limit the study of microstructures, resulting in a sensitivity of only $37 \%$ for the detection of plaque rupture ${ }^{[1]}$. By contrast, OCT uses reflections of infrared light, provides better spatial resolution and faster data acquisition, and uses smaller diameter catheters than IVUS. OCT has high resolution $(>15 \mu \mathrm{m})$ and enables accurate assessment of stent strut positioning and endothelization, and determination of the histological components of coronary plaques (microcalcium deposits and macrophage accumulation) ${ }^{[2-6]}$. However, the low axial penetration of OCT $(1.5-2 \mathrm{~mm})$ fails to provide optimal visualization of the arterial wall, especially in large vessels in which the outer layers of the artery cannot be identified ${ }^{[7]}$. OCT also requires the injection of contrast during imaging. The main limitation of OCT is its inability to produce images in the absence of coronary flow, i.e., complete occlusion caused by thrombosis or dissection. Furthermore, the requirement of contrast in OCT increases the risk of nephropathy in some patients. IVUS and OCT are useful in the study of different coronary diseases. These two methods can be used in a complementary manner to obtain a definitive diagnosis, and help cardiologists select an appropriate invasive strategy.

The patient in our case study had symptoms of accelerated angina at rest for one week. Clinical detection included ECG and cardiac markers, which indicated that the patient had ACS. However, angiography and IVUS failed to find positive culprit lesions or ruptured plaques. In atherosclerotic plaque ruptures, OCT was used to visualize the thin fibrotic cap of the plaque and locate the site of plaque rupture and intimalb tear. Therefore, both OCT and IVUS are useful intravascular imaging methods, each with its advantages and limitations.

OCT is a catheter-based invasive imaging system. Using light rather than ultrasound, OCT enhances imaging resolution that may permit the evaluation of clinical (including diagnostic assessment of coronary atherosclerosis, luminal measurements during PCI, inflammation, lipid necrotic core and thrombus) and research (e.g., fibrous cap thickness and strut level analysis) parameters for the interventional cardiologist. The versatility of the physical properties of light position OCT as an imaging modality could be useful for improving our understanding of the vascular biology of atherothrombosis and assisting in our performance of PCI procedures.

IVUS imaging is the most commonly used method in coronary intervention, but OCT has higher resolution than IVUS. OCT enables a more detailed assessment of certain coronary lesions. As of this writing, no randomized studies are available to confirm the superiority of OCT over IVUS in all cases. However, using both methods in a complementary manner can be helpful for the correct assessment of coronary disease, and aid cardiologists in selecting an optimal therapeutic strategy.

\section{Acknowledgements}

This project was supported by National Key Clinical Specialty and the development health engineering of Jiangsu Province (LJ201116) and Social development of Zhenjiang (SS2012002). 


\section{References}

[1] Bourantas CV, Garg S, Naka KK, et al. Focus on the research util-ity of intravascular ultrasound comparison with other invasivemodalities. Cardiovasc Ultrasound. 2011; 9: 3-5. PMid:21276268 http://dx.doi.org/10.1186/1476-7120-9-2

[2] Carrizo S, Salinas P, Jimenez-Valero S, et al. Utility of opti-cal coherence tomography to assess a hazy intracoronaryimage after percutaneous coronary intervention. Korean Circ J. 2013; 43: 44-47. PMid:23407580 http://dx.doi.org/10.4070/kcj.2013.43.1.44

[3] Regar E, Ligthart J, Bruining N, et al. The diagnosticvalue of intracoronary optical coherence tomography. Herz. 2011; 36: 417-429. PMid:21744151 http://dx.doi.org/10.1007/s00059-011-3487-7

[4] Bouma BE, Tearney GJ, Yabushita H, et al. Evaluation ofintracoronary stenting by intravascular optical coherencetomography. Heart. 2003; 89: 317-320. PMid:12591841 http://dx.doi.org/10.1136/heart.89.3.317

[5] Otake H, Shite J, Ako J, et al. Local determinants of throm-bus formation following sirolimus-eluting stent implantationassessed by optical coherence tomography. JACC Cardiovasc Interv. 2009; 2: 459-466. PMid:19463471 http://dx.doi.org/10.1016/j.jcin.2009.03.003

[6] Kubo T, Imanishi T, Takarada S, et al. Assessment of culpritlesion morphology in acute myocardial infarction: ability of optical coherence tomography compared with intravascularultrasound and coronary angioscopy. J Am Coll Cardiol. 2007; 50: 933-939. PMid:17765119 http://dx.doi.org/10.1016/j.jacc.2007.04.082

[7] Tanimoto T, Imanishi T, Tanaka A, et al. Various types of plaquedisruption in culprit coronary artery visualized by optical coherence tomography in a patient with unstable angina. Circ J. 2009; 73: 187-189. PMid:19001748

http://dx.doi.org/10.1253/circj.CJ-07-0715 